Meta

Journal des traducteurs

Translators' Journal

\title{
Liste des relecteurs 2015
}

Volume 60, numéro 3, décembre 2015

URI : https://id.erudit.org/iderudit/1036132ar

DOI : https://doi.org/10.7202/1036132ar

Aller au sommaire du numéro

Éditeur(s)

Les Presses de l’Université de Montréal

ISSN

0026-0452 (imprimé)

1492-1421 (numérique)

Découvrir la revue

Citer ce document

(2015). Liste des relecteurs 2015. Meta, 60(3), 381-384.

https://doi.org/10.7202/1036132ar

Ce document est protégé par la loi sur le droit d'auteur. L'utilisation des services d'Érudit (y compris la reproduction) est assujettie à sa politique d'utilisation que vous pouvez consulter en ligne.

https://apropos.erudit.org/fr/usagers/politique-dutilisation/
Cet article est diffusé et préservé par Érudit.

Érudit est un consortium interuniversitaire sans but lucratif composé de l’Université de Montréal, l'Université Laval et l'Université du Québec à Montréal. Il a pour mission la promotion et la valorisation de la recherche. https://www.erudit.org/fr/ 


\section{Liste des relecteurs 2015}

Nous remercions chaleureusement toutes celles et ceux qui ont donné de leur temps et qui ont contribué à la revue. Toute omission est involontaire de notre part, veuillez nous la signaler.

Mohammed Alghbban (King Saud University)

Rachele Antonini (Università di Bologna)

Marta Arumí Ribas (Universitat Autònoma de Barcelona)

Paul Bandia (Concordia University)

Allison Beeby (Universitat Autònoma de Barcelona)

Ann Bertels (Katholieke Universiteit Leuven)

Pier-Pascale Boulanger (Concordia University)

Lynne Bowker (Université d'Ottawa/University of Ottawa)

Annie Brisset (Université d'Ottawa/University of Ottawa)

Louise Brunette (Université du Québec en Outaouais)

Joost Buysschaert (Universiteit Gent)

Deborah Cao (Griffith University)

María Calzada Pérez (Universitat Jaume I)

Samantha Cayron (Université de Genève)

José Luís Chamosa González (Universidad de León)

Yung-nan Chiang (National Taipei University of Technology)

Jungwha Choi (Hankuk University of Foreign Studies)

Gyunghee Choi (The University of New South Wales)

Mikyung Choi (Ewha Womans University)

Salvatore Ciancitto (Università degli Studi di Catania)

Gloria Corpas Pastor (Universidad de Málaga)

Gert De Sutter (Universiteit Gent)

Akila Naïma Dib (Université du Québec à Trois-Rivières)

Valérie Dullion (Université de Genève)

Miguel Jesús Duro Moreno (Universidad Autónoma de Madrid)

Roch Duval (Université de Montréal)

Álvaro Echeverri (Université de Montréal)

Elena Ferran Larraz (Universitat Rovira i Virgili)

Cecilia Foglia (Université de Montréal)

Ryan Fraser (Université d'Ottawa/University of Ottawa)

Judit Freixa Aymerich (Universitat Pompeu Fabra)

Chantal Gagnon (Université de Montréal)

Meta LX, 3, 2015 
Anabel Galán-Mañas (Universitat Autònoma de Barcelona)

Lorenzo Gallego Borghini (Universitat Autònoma de Barcelona)

Ramón Garrido Nombela (Universidad de Alcalá)

Jean-Claude Gémar (Université de Montréal)

Anna Ghiglione (Université de Montréal)

Daniel Gile (Université Paris 3 - Sorbonne Nouvelle)

José Luiz Gonçalves (Universidade Federal de Ouro Preto)

Thierry Grass (Université de Strasbourg)

Rainier Grutman (Université d'Ottawa/University of Ottawa)

Zélie Guével (Université Laval)

Iris Guske (Institut für Fremdsprachenberufe Kempten)

Ran HaCohen (Tel Aviv University)

Sonia Halimi (Université de Genève)

María José Hernández Guerrero (Universidad de Málaga)

Iris Angelika Holl (Universidad de Salamanca)

Kaibao Hu (Shanghai Jiao Tong University)

Jaakko Husa (Lapin yliopisto)

Corinne Imhauser (ISTI - Haute École de Bruxelles)

Arnt Lykke Jakobsen (Handelshøjskolen i København)

Laurence Jay-Rayon (Montclair State University)

Meng (Christine) Ji (The University of Sydney)

Miguel Jiménez-Crespo (Rutgers University)

Louis Jolicœur (Université Laval)

Klaus Kaindl (Universität Wien)

Esmaeil Kalantari (Université de Montréal)

Ji-Hae Kang (Ajou University)

Behrouz Karoubi (Universiteit Leiden)

Daejin Kim (Seoul National University of Science and Technology)

Michalis Kokonis (Aristotle University of Thessaloniki)

José Lambert (Katholieke Universiteit Leuven)

Gillian Lane-Mercier (McGill University)

Christopher Larkosh (University of Massachusetts Darthmouth)

Antonio Lavieri (Università Degli Studi Di Palermo)

Sara Laviosa (Università degli Studi di Bari Aldo Moro)

Matthieu Leblanc (Université de Moncton)

Catherine Leclerc (McGill University)

Sungyup Lee (Ewha Womans University)

Benoit Léger (Concordia University)

Clara Inés López Rodríguez (Universidad de Granada)

Kazem Lotfipour-Saedi (Language Assessment and Resource Centre)

Rachel (Wai-chu) Lung (Lingnan University)

Xuanmin Luo (Tsinghua University)

Brigid Maher (La Trobe University)

Elena Maksimova (Duke University)

Carme Mangiron Hevia (Universitat Autònoma de Barcelona)

Maialen Marin-Lacarta (Hong Kong Baptist University)

Elizabeth Marshman (Université d'Ottawa/University of Ottawa) 
Serenella Massidda (Università degli Studi di Sassari)

Anna Matamala (Universitat Autònoma de Barcelona)

Roberto Mayoral Asensio (Universidad de Granada)

Jean-Guy Mboudjeke (University of Windsor)

Christopher Mellinger (Walsh University)

Pierre-Alexis Mével (The University of Nottingham)

Reine Meylaerts (Katholieke Universiteit Leuven)

Marta Miquel Iriarte (Universitat Autònoma de Barcelona)

Sylvie Monjean-Decaudin (Université de Cergy-Pontoise)

Nader Morkus (Indiana University Bloomington)

Jane Moss (Duke University)

Brian Mossop (York University)

Fernando Navarro Domínguez (Universitat d'Alacant)

Robert Neather (Hong Kong Baptist University)

Moses Nyongwa (Université de Saint-Boniface)

Minako O'Hagan (Dublin City University)

Emilio Ortega Arjonilla (Universidad de Málaga)

Nata a Pavlovi (Sveu ili te u Zagrebu)

Luis Pegenaute Rodríguez (Universitat Pompeu Fabra)

Valerie Pellatt (Newcastle University)

Lynn Penrod (University of Alberta)

Éric Poirier (Université du Québec à Trois-Rivières)

Fernando Prieto Ramos (Université de Genève)

Nasrin Rahimieh (University of California Irvine)

Roberta Raine (Lingnan University)

Laurence Raw (Ba kent Üniversitesi)

Michel Richard (Université de Montréal)

Beatriz Rodríguez Rodríguez (Universidade de Vigo)

Sara Rovira-Esteva (Universitat Autònoma de Barcelona)

Dae Young Ryu (Handong Global University)

Myriam Salama-Carr (University of Salford)

Gabriela Saldanha (University of Birmingham)

John Douglas Sanderson (Universitat d'Alacant)

Véronique Sauron (Université de Genève)

Federica Scarpa (Università degli Studi di Trieste)

Kilian Seeber (Université de Genève)

Candace Séguinot (York University)

Adriana Şerban (Université Paul-Valéry Montpellier 3)

Ji-Sun Shin (Ewha Womans University)

Maria Sidiropoulou (National and Kapodistrian University of Athens)

Kenn Nakata Steffensen (University College Cork)

John Stone (Universitat de Barcelona)

Madeleine Stratford (Université du Québec en Outaouais)

Şehnaz Tahir Gürçağlar (Boğaziçi Üniversitesi)

Zaixi Tan (Hong Kong Baptist University)

Kristian Tansgaard Hvelplund (Københavns Universitet)

Maribel Tercedor Sánchez (Universidad de Granada) 
Karen Thornber (Harvard University)

Sonja Tirkkonen-Condit (Itä-Suomen yliopisto)

Cristina Valderrey Reñones (Universidad de Salamanca)

Marc Van Campenhoudt (ISTI - Haute École de Bruxelles)

Piet Van Poucke (Universiteit Gent)

Sylvie Vandaele (Université de Montréal)

Daniel Steve Villarreal (National Defense Medical Center)

Emma Wagner (Commission européenne)

Richard Kelly Washbourne (Kent State University)

Judy Wakabayashi (Kent State University)

Jihong (Lily) Wang (The University of Queensland)

Catherine Way (Universidad de Granada)

Rachel Weissbrod (Bar-Ilan University)

Malcolm Williams (Université d'Ottawa/University of Ottawa)

Zhonghua (Richard) Xiao (Zhejiang University)

Jun Xu (Nanjing University)

Christine York (Concordia University)

Juan Jesús Zaro Vera (Universidad de Málaga) 